\title{
Pleuro-Pulmonary Blastoma: A Rare Cause of Pleural Effusion in a Young Girl
}

\author{
MM RASHID ${ }^{\mathrm{a}}$, MF ALAM ${ }^{\mathrm{b}}$, A HASNAT', ${ }^{\mathrm{Y}}$ ZAMAL $^{\mathrm{d}}$, Z ISLAM ${ }^{\mathrm{e}}$, MM RAHMAN $^{\mathrm{f}}$
}

\begin{abstract}
Summary:
Pleuropulmonary blastoma (PPB) is a rare, primitive primary neoplasm of the thorax that affects children. We present a case of a girl of 11 years who was admitted in National Institute of Diseases of the Chest and Hospital (NIDCH) with the complaints of high grade continued fever and shortness of breath. Initial physical examination and chest radiograph was suggestive of left sided massive pleural effusion. Along with pleural effusion, chest CT depicted a large heterogeneous mass occupying most of the left hemithorax. Computed
\end{abstract}

\section{Introduction:}

Pleuropulmonary blastoma is an aggressive tumor accounting for less than $1 \%$ of all primary malignant lung tumors in the paediatric population ${ }^{1}$. Manivel et al. suggested that PPB is a rare, distinctive intrathoracic/ pulmonary neoplasm, where both the blastematous and sarcomatous components coexist as compared to the typical biphasic epithelial-stromal morphology seen in classic adult type pulmonary blastoma ${ }^{2}$. PPB in children differs from its counterpart in adults because of its variable anatomic location, primitive embryonic-like

a. Dr. Md. Mamunur Rashid, Registrar, Department of Respiratory Medicine, NIDCH, Dhaka.

b. Dr. Mohammad Fakhrul Alam, Medical Officer, Department of Medicine, Comilla Medical College \& Hospital, Comilla, Bangladesh

c. Dr. Md. Abu Hasanat, Assistant Registrar (Respiratory Medicine)

d. Dr. A.K.M. Younus Zamal, Assistant Professor, Department of Pathology, NIDCH, Dhaka.

e. Dr. Md. Zahidul Islam, Assistant Professor, Department of Respiratory medicine,

f. Professor Md. Mostafizur Rahman, Ex-Director \& Head of the Department, NIDCH, Dhaka.

Address of correspondence: Dr. Md. Mamunur Rashid, MBBS, FCPS (Medicine), MD (Chest), Registrar, Department of Respiratory Medicine, National Institute of Diseases of the Chest and Hospital, Dhaka, Bangladesh, Mobile no: +8801711903377 , E-Mail: dr.mamun98@yahoo.com

Received: 12 February, 2011

Accepted: 25 April, 2012 tomography and bone scintigraphy suggested the mass to have a primary pulmonary origin. Cytologically the lesion was consistent with pleuropulmonary blastoma. Due to pleural involvement and presence of large inoperable mass, we considered chemotherapy as the first modality of treatment prior to surgery and managed thereby.

Key wards: Pleuropulmonary blastoma, pleural effusion, pulmonary blastoma of childhood

(J Bangladesh Coll Phys Surg 2012; 30: 173-176)

blastema and stroma, absence of a carcinomatous component and potential for sarcomatous differentiation ${ }^{2}$. We herein report a case of PPB (suggestive of type III), presenting with radiological feature of large mixed density pulmonary mass with pleural effusion ${ }^{2}$.

\section{Presentation of the case:}

An 11-year-old girl, student of class-VIII was admitted in NIDCH on $12^{\text {th }}$ July, 2008 with fever and shortness of breath. She developed high grade continued fever and cough productive of mucoid sputum for two weeks. She experienced heaviness of the left side of the chest and progressively worsening respiratory distress for about 10 days. At the time of presentation, she used to become breathless while walking on the flat surface at her own pace at close distances and developed inability to lie down on right lateral position. She was in her junior high school study. She had no significant past medical history and there was no history of tumors in her close relatives.

She was a thin, alert, young girl with preferred left lateral decubitus. Her temperature was 101ÚF, pulse was 110 beats per minute, respiratory rate was 26 breaths per minute, blood pressure was 100/70 mm Hg, and her oxygen saturation was $96 \%$ while she was breathing room air. She had no cyanosis, clubbing, lymphadenopathy or bony tenderness. Trachea was shifted towards the right side and apex beat was impalpable. The left hemithorax was immobile with dull 
on percussion and absent breath sound, both anteriorly and posteriorly. The remainder of the physical examination was normal.

Complete blood count revealed the hemoglobin level was $8.8 \mathrm{~g} / \mathrm{dl}$, erythrocyte sedimentation rate was 105 $\mathrm{mm}$ in $1^{\text {st }}$ hour. She had a negative tuberculin skin test. Staining for acid fast bacillus was negative on three sputum specimens as was Gram's staining. Chest radiograph showed an apparent left sided pleural effusion with mediastinal shift to the right side.

A thoracocentesis was performed according to ATS guideline and removed about $1000 \mathrm{ml}$ of haemorragic fluid which was exudative, lymphocyte predominant without showing any AFB and malignant cell. Pleural biopsy was taken by Abrahm's needle but failed to detect any granulomatous or malignant lesion. Fibre-optic broncoscopy revealed no endobronchial growth, but left lower lobe bronchus was narrowed due to external compression. Bronchial washings were negative for malignant cells. After 3 days, patient developed severe respiratory distress and left chest drainage was given.

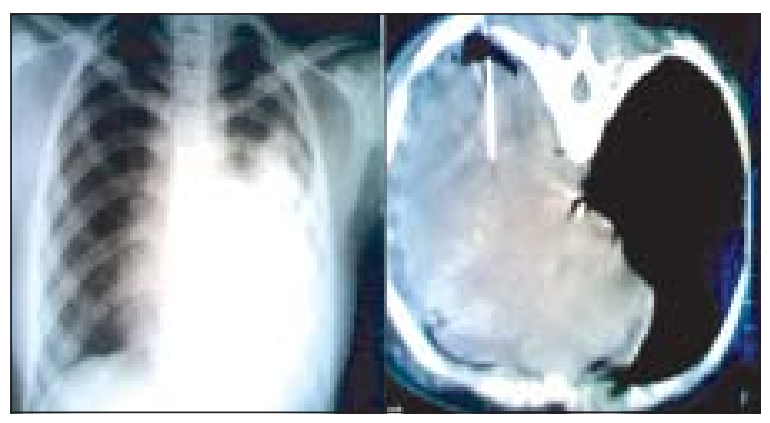

Fig.1. (A): Chest X-Ray P/A view with a mass lesion involving the left upper and mid zone. (B) CT scan of chest showing a large mixed density mass in the left lung medially merging with the mediastinum, in situ FNAC needle is also seen.

Repeat chest X-Ray after thoracocentesis revealed a mass lesion involving the left upper and mid zone (Figure 1A). Computed tomography (CT) scanning of thorax was performed for further delineation of the lesion. A large mixed density mass lesion was seen in the left lung medially merging with the mediastinum. (Figure 1B) Small left sided pleural effusion was also noted. There was no hilar or mediastinal lymphadenopathy.

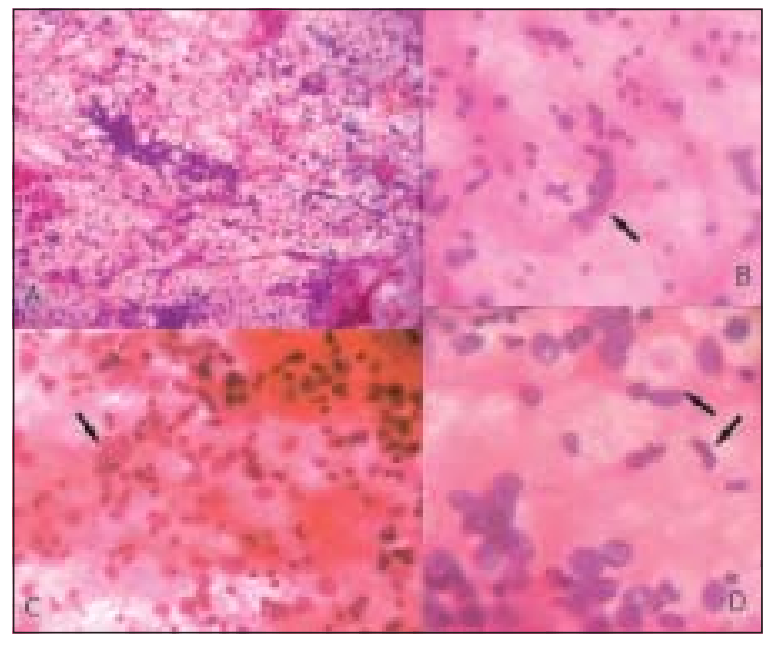

Fig.2. (A): Light microscopy of the smear shows cluster of primitive appearing cells. $(H \& E, \times 100)(B) \&(C)$ Group of small primitive appearing cells having round to oval nuclei and scanty cytoplasm. $(H \& E, \times 400)(D)$ Arrow shows individually dispersed spindle shaped cells with elongated nuclei. $(H \& E, \times 400)$

A CT guided FNAC was performed. Smear showed group of small primitive appearing cells having scanty cytoplasm with indistinct borders and round to oval nuclei with finely granular, evenly distributed hyperchromatic chromatin and inconspicuous nucleoli; on a background of necrotic material. (Figure 2A-C) A less frequent second cellular population consisted of individually dispersed, elongated or spindle-shaped cells with similar-appearing nuclei was also found.(Figure 2D) There was no evidence of neoplastic epithelium within the smears. Morphologically this finding is quite consistent with PPB.

Presence of metastasis was verified by whole body bone scan and MRI of brain, but there was no active lesion. Ultrasonography of abdomen also revealed no abnormality. Due to presence of inoperable huge tumor, after consulting with thoracic surgeons, patient was referred to medical oncologists for further management.

\section{Discussion:}

Pleuropulmonary blastoma is a rare malignant dysontogenetic neoplasm primarily affecting children ${ }^{3}$. It was previously known as cystic rhabdomyosarcoma, pulmonary blastoma of childhood or rhabdomyosarcoma arising in a cystic adenomatoid malformation. This neoplasm occurs not only in lung, 
but also may arise from mediastinum, diaphragm and/ or pleura 4 . PPB is classified into three categories by gross and microscopic examination - type I (purely cystic), type II (cystic and solid) and type III (purely solid) $)^{5}$. These three types of PPB are presumed to have histogenetic linkage with the potentials of progression into other forms ${ }^{3}$. The PPB in the present case has predominantly solid blastematous component and belongs to the type III subcategory.

Pleuropulmonary blastoma occurs almost exclusively in children under the age of 12 years $^{6}$, usually by the age of 4 years, with a median age of 2 years $^{7}$. Type I tumours occur in the youngest children, type III in the oldest ${ }^{8}$. This is consistent with our recent case. The lower lobe of the lung is most commonly involved than the upper and middle lobe. The disease is usually unilateral, although bilateral lesions have been reported 5 .

According to the clinic-pathological study conducted by Priest et al, the commonest clinical presentation is respiratory distress ${ }^{8}$, as seen in the present case. Other symptoms include fever, cough, chest or abdominal pain, malaise and anorexia ${ }^{5}$. A suspected pulmonary infection is the most frequent clinical suspicion in these patients. Radiographically, type III PPB is typically a heterogeneous solid mass with or without involvement of chest wall or mediastinal structures. The entire hemithorax may be opacified by the mass. We have similar radiological finding in our case.

Histologically, PPB is characterized by a biphasic neoplastic population of primitive appearing small round cells and larger elongated or spindle-shaped sarcomatous elements, but a malignant epithelial component does not occur ${ }^{9}$, a key morphologic point in differentiating PPB from the adult pulmonary blastoma. A proportion of these cancers may also manifest rhabdomyosarcomatous, chondrosarcomatous or liposarcomatous differentiation ${ }^{4}$. Immunohistochemical positivity with vimentin, muscle-specific actin, desmin, S-100 protein etc. can strengthen the diagnosis in such a case $\mathrm{e}^{10}$.

The cytopathologic findings of PPB may represent some or all of its histopathologic components ${ }^{9}$. In our case, the two major cell types-the primitive blastemal elements and spindle-shaped cells-were represented in the aspiration smears. But there was no recognizable differentiation in the blastemal or mesencymal component, also any evidence of neoplastic epithelium within the smears. All together goes in favour of PPB.

A significant feature is that up to $25 \%$ of patients with PPB or their young relatives have associated dysplastic or neoplastic lesions, such as pulmonary cyst, cystic adenoid malformation, cystic nephroma, medulloblastoma, and thyroid neoplasia; this is called the PPB family cancer syndrome. ${ }^{1,11}$ In our patient there was no such positive history. Several cytogenetic studies have shown the detection of numerical and structural chromosomal abnormalities of 2 and 8 in isolated cases. ${ }^{3,12}$ Thus, once the diagnosis of PPB is made, a thorough search for tumors should be initiated in the close relatives.

For the metastatic propensity, the CNS metastasis rate was $44 \%$ of all recurrences in the study of 50 cases of PPB by Priest et al, and patients with pleural or mediastinal involvement fared significantly worse than those without such involvement ${ }^{8}$. Other common metastatic sites include bone, lymph nodes, liver, pancreas, kidney, and adrenal glands ${ }^{4}$. Presence of pleural effusion suggests involvement of pleura in our patient, although evidence for distant metastasis was absent.

Complete tumor ablation is essential to prevent local recurrence and for survival, so the main goal of therapy should be radical surgery, followed by chemotherapy. Some authors suggest that chemotherapy should be given with local radiotherapy in the majority of type II and type III patients ${ }^{10,13}$ Unfortunately our patient was an inoperable one and we referred the patient for chemotherapy.

There is a better outcome of type I PPB compared to the other two types; most type I PPB patients have 80$90 \%$ five year disease-free survival ${ }^{10}$. At present, type II and III patients have less than 50\% five year survival ${ }^{10}$. Like our patient, those with pleural, mediastinal or extrapulmonary involvement at the time of diagnosis have worse prognosis than others without such involvement ${ }^{13}$. 


\section{Reference:}

1) Dishop M K and Kuruvilla S. Primary and Metastatic Lung Tumors in the Pediatric Population: A Review and 25-Year Experience at a Large Children's Hospital. Archives of Pathology \& Laboratory Medicine: 2008, 132, 7, 1079-1103.

2) Bhasin T S, Bhalla A, Mannan R et al. Cytology of Pleuropulmonary Blastoma: A Case Report. Journal of Clinical and Diagnostic Research. 2011 ,5: 1656-1658.

3) Lee CH, Kim KI, Kim YD, et al. Pleuropulmonary Blastoma in a Young Adult Presenting as a Ruptured Cystic Teratoma in Radiology. J Korean Med Sci 2003; 18: 595-8.

4) Abdelmohsen MH, Serag AR, Bayoumi SR. Pleuropulmonary Blastoma: A Case Report. The Internet Journal of Thoracic and Cardiovascular Surgery. 2004. Volume 6 Number 2. Available from: URL: http://www.ispub.com/entrez/query/ fcgi.htm

5) Leung J, Tsao J, Lai AKH, et al. Pleuropulmonary blastoma in an infant presenting with tension pneumothorax. JHK Coll Radial 2001; 4:219-21.

6) Vargas SO, Nose V, Fletcher JA, et al. Gains of chromosome 8 are confined to mesenchymal components in pleuropulmonary blastoma. Pediatr Dev Pathol 2001; 4:434-45.
7) Federici S, Domenichelli V, Tani G, et al. Pleuropulmonary blastoma in congenital cystic adenomatoid malformation: report of a case. Eur J Pediatr Surg 2001; 11:196-9.

8) Priest JR, McDermott MB, Bhatia S, et al. Pleuropulmonary blastoma: a clinicopathologic study of 50 cases. Cancer 1997; 80:147-61.

9) Kathleen K, Kim R. The cytomorphology of pleuropulmonary blastoma. Arch Pathol Lab Med 2000; 124:416-18.

10) Hill DA, Jarzembowski JA, Priest JR, et al. Type I pleuropulmonary blastoma: pathology and biology study of 51 cases from the international pleuropulmonary blastoma registry. Am J Surg Pathol 2008; 32:282-95.

11) Kusafuka T, Kuroda S, Inoue M, et al. P53 gene mutations in pleuropulmonary blastomas. Pediatr Hematol Oncol 2002; 19:117-28.

12) Quilichini B, Andre N, Bouvier C, et al. Hidden chromosomal abnormalities in pleuropulmonary blastomas identified by multiplex FISH. BMC Cancer 2006; 6:4-8.

13) Indolfi P, Casale F, Carli M, Bisogno G, Ninfo V, Cecchetto $\mathrm{G}$, et al. Pleuropulmonary blastoma: management and prognosis of 11 cases. Cancer 2000; 89:1396-401. 